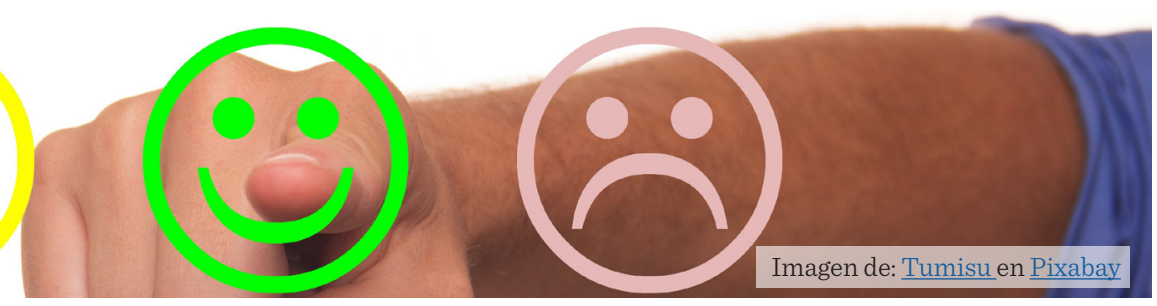

\title{
La relación entre la calidad en el servicio, satisfacción del cliente y lealtad del cliente: un estudio de caso de una empresa comercial en México
}

\section{The relationship between service quality, customer satisfaction, and customer loyalty: A case study of a trading company in Mexico}

\author{
Juan Gilberto Silva-Treviño' ${ }^{1}$ Bárbara Azucena Macías-Hernández²*, Edgar Tello-Leal², Jesús Gerardo Delgado-Rivas
}

\section{RESUMEN}

La calidad en el servicio se considera una alternativa para que las empresas puedan obtener una ventaja competitiva y sostenible en un entorno económico globalizado. Las pequeñas y medianas empresas deben ofrecer una mayor calidad en el servicio que las empresas grandes, y así obtener la preferencia de los clientes. El objetivo de este estudio fue identificar la relación entre la variable calidad en el servicio y las variables satisfacción del cliente y lealtad del cliente. Se utilizó el coeficiente de correlación de Spearman y un método estadístico basado en análisis factorial exploratorio que apunta a extraer la varianza máxima del conjunto de datos dentro de cada factor. Los resultados permitieron observar una correlación altamente significativa, positiva y fuerte de la variable de calidad en el servicio con satisfacción del cliente $(r=0.820)$ y lealtad del cliente $(r=0.803)$. Un hallazgo importante también fue la asociación entre la dimensión aspectos tangibles con las variables satisfacción del cliente $(r=0.910)$ y lealtad del cliente $(r=0.919)$. Por otro lado, en el análisis factorial, a través de la varianza total explicada, se observó que el autovalor es superior a 1 en los cinco primeros casos, donde el porcentaje de la varianza alcanza un valor máximo de $54.886 \%$ en su primer factor. Entonces, con cinco factores se consigue explicar un $73.713 \%$ de la varianza de todos los datos originales. El estudio presentó la limitación de su aplicación en solo una empresa. Se confirmó que a través de una mejor atención y servicio al cliente, la calidad en el servicio constituye una excelente herramienta para la rentabilidad y sostenibilidad de la empresa.

PALABRAS CLAVE: calidad en el servicio, satisfacción del cliente, lealtad del cliente, PyME.

\section{ABSTRACT}

Service quality is considered an alternative for companies to obtain a competitive and sustainable advantage in a globalized economic environment. Small and medium-sized enterprises must offer a higher quality of service than large companies, and thus obtain customer preference. The objective of this study was to identify the relationship between service quality variable and the customer satisfaction and customer loyalty variables. Spearman's correlation coefficient was used, and a statistical method based on exploratory factor analysis, aiming to extract the maximum variance of the data set within each factor. The results allowed observing a highly significant, positive, and strong correlation, with values of $r=0.820$ and $r=0.803$, between the variables of service quality, customer satisfaction, and customer loyalty. An important finding was the association between the tangible aspects dimension with the customer satisfaction and customer loyalty variables, with values of $r=0.910$ and $r=0.919$, respectively. On the other hand, in the factor analysis, through the explained total variance, it was observed that the eigenvalue is greater than 1 in the first five cases, where the percentage of the variance reaches a maximum value of $54.886 \%$ in its first factor. Then, with five factors, $73.713 \%$ of the variance of all the original data is explained. The study presented the limitation of its application in only one company. It was confirmed that, through better customer care and service, service quality constitutes an excellent tool for the profitability and sustainability of the organization.

KEYWORDS: service quality, customer satisfaction, customer loyalty, SME.

*Correspondencia: bmaciash@docentes.uat.edu.mx/Fecha de recepción: 19 de septiembre de 2020/Fecha de aceptación: 5 de octubre de 2020/ Fecha de publicación: 30 de enero de 2021.

${ }^{1}$ Universidad Autónoma de Tamaulipas, Facultad de Comercio y Administración-Victoria, Ciudad Victoria, Tamaulipas, México. ${ }^{2}$ Universidad Autónoma de Tamaulipas, Facultad de Ingeniería y Ciencias, Centro Universitario Victoria, Ciudad Victoria, Tamaulipas, México, C. P. 87149. 


\section{INTRODUCCIÓN}

出 Actualmente, en un entorno económico globalizado, las empresas requieren elevar sus índices de eficiencia y competitividad para conseguir la preferencia de los clientes. En este sentido, la calidad en el servicio es una alternativa para que las empresas puedan obtener una ventaja única y sostenible respecto a sus competidores, independientemente de la actividad comercial o de los servicios que ofrezcan (Azman y Yusrizal, 2016; Shah y col., 2018). La "calidad en el servicio" se entiende como la brecha que existe entre las expectativas del cliente (lo que quiere) y sus percepciones (lo que obtiene) después de recibir un servicio (Bustamante, 2015; Jain y Aggarwal, 2017; Lai y Nguyen, 2017). Por un lado, cuando una empresa cumple con las expectativas del cliente, se consolida la "satisfacción del cliente" (Murali y col., 2016). Por otro lado, cuando un cliente se encuentra en un estado de "satisfacción del cliente" tiende a repetir su comportamiento de compra, generando un estado de "lealtad del cliente" (Ahrholdt y col., 2017). Por tanto, la satisfacción y lealtad del cliente mantienen un vínculo fuerte.

La lealtad del cliente constituye uno de los mejores indicadores para medir el éxito (Nyadzayo y Knajehzadeh, 2016) y rentabilidad de una empresa (Sandada y Matibiri, 2016; Srivastava y Rai, 2018). Se considera que incrementar un $5 \%$ la tasa de lealtad del cliente posibilita que las utilidades puedan aumentar entre $25 \%$ a $45 \%$ (Srivastava y Rai, 2013; Sandada y Matibiri, 2016). Entonces, al compatibilizar con el cliente se obtiene su satisfacción y se elevan las utilidades para la empresa. Al respecto, estudios previos señalan que atraer un nuevo cliente cuesta entre cinco y seis veces más que mantener a uno de la cartera actual (López y Díaz, 2012). En resumen, se puede interpretar que existe una relación entre cuatro conceptos: 1) calidad en el servicio, 2) satisfacción del cliente, 3) lealtad del cliente y 4) utilidades. Al incrementar el nivel de la calidad en el servicio aumentará el indicador de satisfacción del cliente (Kasiri y col., 2017; El-Adly, 2019). Por tanto, al elevar ambos indicado- res, la lealtad del cliente se incrementará y por consecuencia aumentarán las utilidades (Azman y Gomiscek, 2015; Shah y Baloch, 2017). El conocer los requerimientos del cliente le permite a la empresa anticiparse para cumplir sus expectativas en la entrega de un servicio o un producto (Murali y col., 2016; Malhotra y col., 2018).

La calidad en el servicio y su relación con la satisfacción y lealtad del cliente, es un aspecto muy importante en la operación de las Pequeñas y Medianas Empresas (PyME) (Aznar y col., 2016; Usman y col., 2019; Guatzozón y col., 2020). Estas ocupan un lugar preponderante en la economía tanto de los países emergentes como de los desarrollados (Ferreira-deAraújo y col., 2019), representando más de la mitad del empleo formal a nivel mundial, y contribuyen en promedio entre $50 \%$ y $60 \%$ del Producto Interno Bruto (PIB) en los países miembros de la Organización para la Cooperación y Desarrollo Económico (OCDE, 2018). En Estados Unidos, las pymes producen $46 \%$ del PIB y dan empleo a más de 58 millones de personas, de acuerdo con la Administración de Pequeños Negocios (SBA, por sus siglas en inglés: Small Business Administration) (SBA, 2017). En México, las pymes conforman un pilar fundamental en la economía nacional al generar $75 \%$ del empleo, según el Instituto Nacional de Estadística y Geografía (INEGI, 2015). Los criterios utilizados comúnmente para clasificarlas son los siguientes: número de trabajadores, total de ventas anuales, ingresos y activos fijos, entre otros (Banwo y col., 2017; INEGI, 2019). En México se clasifican, de acuerdo con el número de empleados: en micro, pequeña y mediana empresa, y según al sector económico al que pertenece: comercio, manufacturas y servicios (INEGI, 2019).

El ciclo de vida de una empresa tipo pyme puede ser muy corto. En Estados Unidos aproximadamente un $30 \%$ fracasa durante los primeros dos años de vida, de acuerdo con información de la Oficina de estadísticas laborales (BLS, por sus siglas en inglés: Bureau of Labor Statistics) (BLS, 2016). En México, la probabili- 
dad de que una pyme con sólo un empleado sobreviva el primer año es del $62 \%$, y con una esperanza de vida de aproximadamente 7.7 años (INEGI, 2015). Esto significa que su supervivencia constituye uno de sus principales retos, por lo que debe hacer todo lo posible para mantener a los clientes y llegar a una posición en la que ellos mismos divulguen comentarios positivos de la empresa (Salinas-Reyes y col., 2018). En las pymes supervivientes se identifican ciertos factores para mantener a la empresa rentable, tales como la satisfacción y lealtad del cliente por la prestación de un servicio o de un producto (Michna, 2018).

Algunos autores han estudiado la calidad en el servicio mediante los instrumentos Servperf (del inglés: Service Performance) y Servqual (del inglés: Service Quality), que se utilizan constantemente en este tipo de investigaciones (Ibarra-Morales y Casas-Medina, 2015; Nyadzayo y Knajehzadeh, 2016; Murali y col., 2016; Saleem y col., 2017; Palese y Usai, 2018; Shah y col., 2018). El modelo Servperf se conforma de los mismos ítems y dimensiones que utiliza Servqual, pero elimina la parte que hace referencia a las expectativas de los clientes (Cronin y col., 1994; Ibarra-Morales y Casas-Medina, 2015), enfocándose en la medición y evaluación de la percepción del cliente con respecto a la calidad en el servicio.

Kumar y Hundal (2019) evaluaron la calidad en el servicio de una empresa de servicios de energía solar, y encontraron que la brecha entre la expectativa y la percepción del cliente es mínima en las dimensiones de confiabilidad, responsabilidad, confianza y empatía. También, Lai y Nguyen (2017) estimaron la calidad en el servicio a usuarios de una empresa de telecomunicaciones, detectando que las dimensiones de empatía, confianza y aspectos tangibles son las que influyen fuertemente en la satisfacción del cliente. Por otro lado, Ibarra-Morales y Casas-Medina (2015) midieron la calidad en el servicio en una empresa de telefonía celular, y observaron que las dimensiones aspectos tangibles, confianza y empatía inciden fuertemente en la satisfacción del cliente.
Los comentarios sobre la satisfacción del cliente deben recopilarse regularmente, y si se usan correctamente pueden ser invaluables para construir relaciones y lealtad del cliente (Blut y col., 2015; Kasiri y col., 2017). Las empresas que no reúnen información sobre la calidad en el servicio, satisfacción y lealtad del cliente no saben si están en el camino correcto o dónde necesitan adaptar su oferta para satisfacer las necesidades de sus consumidores (Fourie, 2015). Lo anterior es importante para las pymes, ya que no se pueden permitir perder a sus clientes, por el alto costo que implica adquirir nuevos.

El objetivo de esta investigación fue identificar la percepción que tienen los clientes con respecto a la prestación del servicio de una empresa clasificada como pyme considerada líder en su ramo, evaluando la relación de la calidad en el servicio con la satisfacción y lealtad del cliente.

\section{MATERIALES Y MÉTODOS}

En el desarrollo del estudio se aplicó un método de investigación conocido como "Estudio de caso" (Yin, 2013). Un estudio de caso riguroso puede ser conducido con propósitos de exploración, descripción o explicación (Sarmiento y col., 2018). El enfoque propuesto en la presente investigación es de naturaleza exploratoria y descriptiva, además de exhibir un alcance correlacional, entre dos o más variables.

Se definió un modelo estructurado de investigación tal como se muestra en la Figura 1, en el que se presentan tres variables dominantes del estudio: calidad en el servicio, satisfacción del cliente y lealtad del cliente. Además, la calidad en el servicio se conforma de cinco dimensiones: tangibles, confiabilidad, responsabilidad, confianza y empatía (Guesalaga y Pitta, 2014), las cuales se consideran variables en este estudio.

La dimensión aspectos tangibles está representada por los elementos relacionados con la apariencia de los empleados y de la empresa. Eva- 


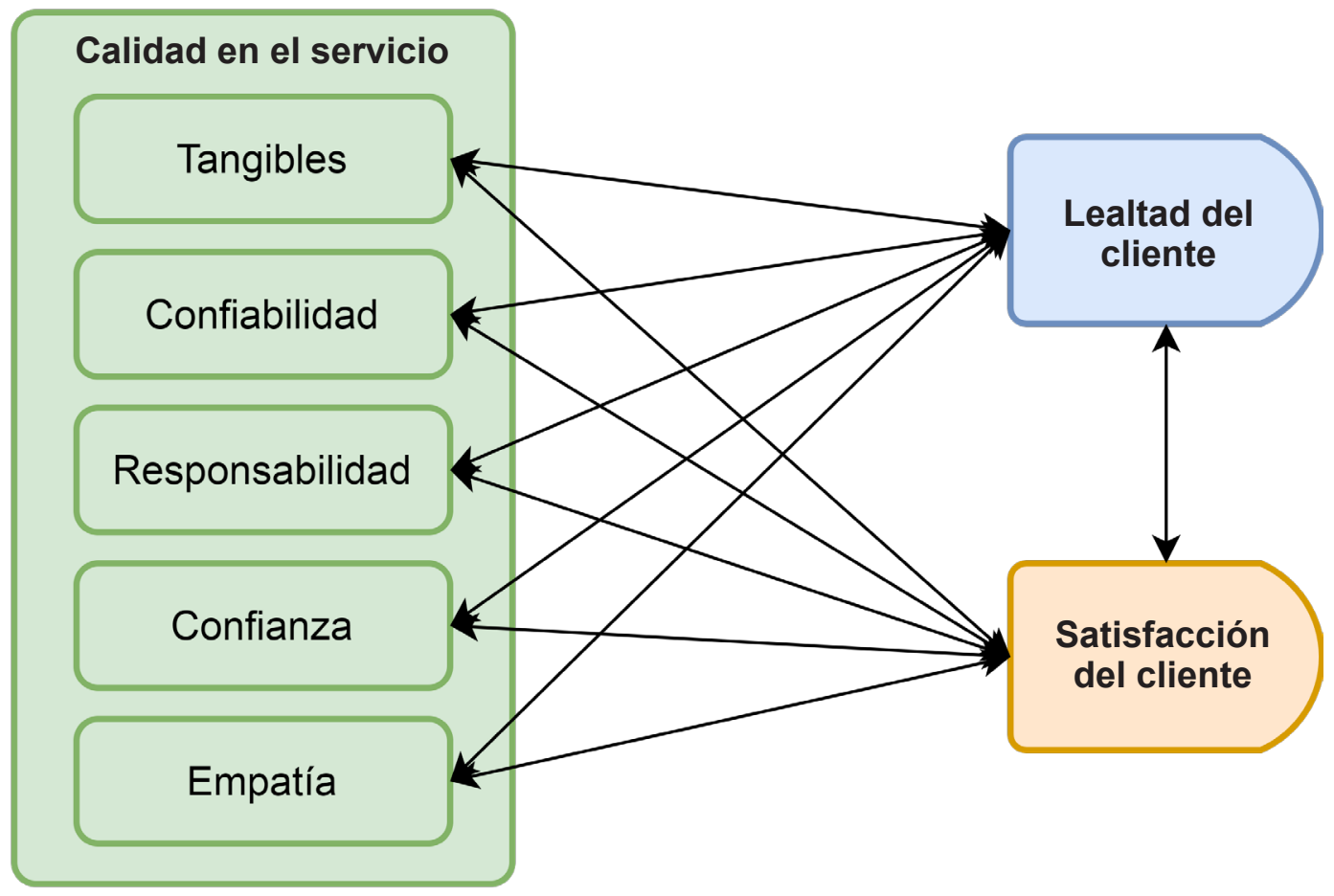

Figura 1. Variables que se estudian en el modelo de investigación.

Figure 1. Variables studied in the research model.

lúa la apreciación de los clientes, en primera instancia, con respecto al entorno físico de la organización, es decir, el equipo utilizado para prestar el servicio y el diseño de la construcción (Murali y col., 2016). La dimensión confiabilidad consiste en las capacidades de la empresa para cumplir de forma precisa y confiable con los servicios que ofrece y la disposición de los empleados para brindar el servicio (Zhang y Hou, 2013). La dimensión responsabilidad evalúa la atención de los empleados con respecto al deseo y voluntad de apoyar a los clientes, así como proporcionar un servicio rápido y eficiente (Nyadzayo y Khajehzadeh, 2016). La dimensión confianza considera la seguridad que los empleados transmiten a los clientes a través de los conocimientos y habilidades del servicio prestado, además de la cortesía en la atención, aspecto profesional, competencia técnica y comportamiento interpersonal (Murali y col., 2016). Finalmente, la dimensión empatía se vincula con el cuidado y atención personalizada que la empresa proporciona, considerando aspectos co- mo horarios de servicio y políticas de atención al cliente (Zhang y Hou, 2013).

\section{Recopilación de datos}

El estudio de caso se llevó a cabo en una empresa del ramo papelero, con más de 30 años de operación en Ciudad Victoria, Tamaulipas, considerada líder en la distribución y servicio de papelería y artículos de oficina, que se caracteriza por ofrecer precios de mayoreo para el público en general. Además, ha incorporado artículos de electrónica, cómputo y consumibles para equipo de cómputo. La empresa se clasifica dentro del tipo pyme como mediana empresa del sector comercio (SE, 2009), por el total de empleados, que son 93, y por el monto de ventas anuales, considerando las de 2019. En la primera entrevista con el gerente se identificaron las siguientes características de la empresa: 1) el modelo de negocio implementado opera como proveedor mayorista para un grupo de pymes del mismo giro comercial en la región y un área de ventas para atención exclusiva a empresas, así 
como la venta directa en mostrador; 2) crecimiento regional (en los últimos 5 años), es decir, apertura de sucursales en las ciudades del estado con mayor población; 3) competitividad local, considerando una estructura organizacional basada en un plan de crecimiento y procesos definidos para la operación del negocio; 4) ventas con enfoque de comercio electrónico y de servicio de entrega a domicilio.

La recolección de datos se llevó a cabo mediante un cuestionario conformado por tres secciones y 34 ítems en total (Tabla 1). La primera sección consta de 5 ítems que tienen como objetivo identificar los datos demográficos básicos de los encuestados. La segunda se compone de 22 ítems que permiten conocer la experiencia de los clientes con respecto a la clase de servicio que le proporciona la empresa. La tercera está integrada por 7 ítems cuyo objetivo es valorar la satisfacción y lealtad del cliente con respecto a la prestación del servicio (Tabla 1). La segunda y tercera sección del cuestionario se construyeron utilizando una escala Likert, que se conforma de 5 puntos para medir la percepción del cliente. El valor menor en la escala corresponde a "Muy en desacuerdo" y el punto más alto indica "Muy de acuerdo".

La segunda sección habilita la recopilación de información de la variable calidad en el servicio, basada en el modelo Servperf para la valoración del desempeño (Cronin y col., 1994), el cual se fundamenta en el modelo Servqual (Parasuraman y col., 1988). La tercera sección permite evaluar las variables satisfacción del cliente y lealtad del cliente. Esta sección se conforma de 3 ítems para recolectar percepciones relacionadas con la primera variable y 4 ítems para la segunda (Tabla 2). Los ítems de la variable lealtad del cliente se basan en la investigación realizada por Shi y col. (2014). Los ítems definidos para la variable satisfacción del cliente se basan en el modelo del Índice de Satisfacción del Cliente Estadounidense (ACSI, por sus siglas en inglés: American Customer Satisfaction Index) (ACSI, 2018). El puntaje de ACSI se actualiza trimestralmente y considera datos de 10 sectores económicos y 46 industrias.

Para el diseño y la aplicación del instrumento (segunda y tercera sección) se realizó su traducción del idioma inglés a español, ajustando los ítems al contexto de aplicación (en la Tabla 2 se muestran los ítems contenidos en el instrumento). Se validó la traducción con un especialista en el idioma inglés, con conocimiento en el tema de investigación; no se realizó prueba piloto en el proceso de aplicación del cuestionario y no fue necesario realizar cambios en la redacción de las preguntas o estructura de las respuestas, a excepción de la primera sección de datos demográficos, en la que se tomaron los aportados por la dirección general.

\section{Condiciones del muestreo}

A la dirección general de la empresa analizada en el presente trabajo, se le hizo el planteamiento verbal del objetivo, propósito, alcance y utilidad del estudio. A continuación, de forma conjunta, el director general y los investigadores revisaron el contenido de cada una las secciones que integran el instrumento.

Tabla 1. Estructura del cuestionario.

Table 1. Questionnaire structure.

\begin{tabular}{|l|c|c|c|c|}
\hline \multirow{2}{*}{} & Datos generales & \multicolumn{2}{|c|}{ Percepción del cliente } \\
\cline { 2 - 5 } & Sección I & Sección II & \multicolumn{2}{|c|}{ Sección III } \\
\cline { 2 - 5 } & Perfil socioeconómico & Calidad en el servicio & Satisfacción del cliente & Lealtad del cliente \\
\hline Ítems & 5 & 22 & 3 & 4 \\
\hline
\end{tabular}


Tabla 2. Ítems contenidos en el instrumento utilizando en el estudio.

Table 2. Items contained in the instrument used in the study.

\begin{tabular}{|c|c|}
\hline Sección & Ítem \\
\hline \multirow{4}{*}{ Tangibles } & 6. Los equipos que se utilizan dentro de la papelería son modernos \\
\hline & 7. Las instalaciones en la papelería son visualmente atractivas \\
\hline & 8. Los empleados de la papelería tienen buena presentación \\
\hline & 9. La papelería está ordenada y limpia \\
\hline \multirow{5}{*}{ Confiabilidad } & $\begin{array}{l}\text { 10. Se cumplen con los tiempos de espera cuando solicita algún servicio dentro de la } \\
\text { papelería }\end{array}$ \\
\hline & 11. Cuando usted tiene un problema el personal muestra interés genuino en resolverlo \\
\hline & 12. Los trabajadores proporcionan un buen servicio a la primera \\
\hline & 13. La atención al cliente dentro de la papelería se realiza en un tiempo razonable \\
\hline & 14. Se esfuerzan para no cometer errores en el servicio \\
\hline \multirow{4}{*}{ Responsabilidad } & 15. El cliente sabe exactamente el tiempo en que tardarán en proporcionar el servicio \\
\hline & $\begin{array}{l}\text { 16. El servicio en la papelería con relación al número de clientes es relativamente } \\
\text { rápido }\end{array}$ \\
\hline & 17. Los trabajadores de la papelería siempre están dispuestos a proporcionar ayuda \\
\hline & 18. Los trabajadores de la papelería atienden las solicitudes de los clientes rápidamente \\
\hline \multirow{4}{*}{ Confianza } & 19. Los trabajadores inspiran confianza a los clientes \\
\hline & $\begin{array}{l}\text { 20. Te sientes seguro y tranquilo cuando interaccionas con los trabajadores de la } \\
\text { papelería }\end{array}$ \\
\hline & 21. Los empleados son educados y corteses \\
\hline & 22. Los empleados de la papelería están capacitados \\
\hline \multirow{5}{*}{ Empatía } & 23. La papelería brinda atención personalizada \\
\hline & 24. Los trabajadores de la papelería brindan atención personal \\
\hline & 25. Los trabajadores de la papelería atienden sus necesidades específicas \\
\hline & 26. Los trabajadores muestras interés genuino para atender \\
\hline & 27. Las horas de servicio que la papelería ofrece son adecuadas \\
\hline \multirow{3}{*}{$\begin{array}{l}\text { Satisfacción del } \\
\text { cliente }\end{array}$} & 28. En general me encuentro satisfecho con el servicio que la papelería brinda \\
\hline & 29. La atención que brinda la papelería excede mis expectativas \\
\hline & 30. El servicio en la papelería excede mis expectativas \\
\hline \multirow{4}{*}{$\begin{array}{l}\text { Lealtad del } \\
\text { cliente }\end{array}$} & 31. Tengo la intención de continuar asistiendo a la papelería en los próximos años \\
\hline & 32. Voy a recomendar la papelería a aquellas personas que me pregunten mi opinión \\
\hline & 33. Voy a decir cosas positivas de la papelería \\
\hline & $\begin{array}{l}\text { 34. Prefiero acudir a esta papelería aun si otra papelería ofreciera el mismo trato o } \\
\text { servicio }\end{array}$ \\
\hline
\end{tabular}

Posteriormente, el director autorizó la asignación de un espacio dentro de las instalaciones de la empresa (matriz) cercano al área de "cajas", a fin de que el cliente encontrara un ambiente cómodo y agradable al recibir la propuesta para participar en el estudio y responder el instrumento.
Un total de 129 cuestionarios fueron aplicados del 10 al 28 de junio de 2019, con una tasa de participación de aproximadamente $25 \%$, consultándose en total a 516 clientes. El levantamiento del cuestionario se llevó a cabo por medio de 2 encuestadores previamente capacitados, de lunes a viernes en 3 horarios: de 
9:00 a 11:00 a. m., de 1:00 a 3:00 p. m. y de 5:00 a 7:00 p. m. Los encuestadores invitaron a los clientes a participar en la encuesta después de concluir su proceso de compra. A los que aceptaron participar en el estudio se les proporcionó el cuestionario en formato impreso y los encuestadores solamente apoyaron en caso de que se presentara alguna duda sobre los ítems o su llenado.

\section{Análisis estadístico}

Con la finalidad de verificar la confiabilidad de la información recopilada, se calculó el coeficiente alfa de Cronbach para las tres variables de la investigación y para las dimensiones de la variable calidad en el servicio. Posteriormente, el análisis estadístico de los datos se llevó a cabo a través del cálculo de la matriz de correlación basada en el coeficiente de Spearman, la cual permite identificar la asociación entre las variables. En este caso, se utilizó el coeficiente de Spearman debido a la distribución no paramétrica de la que proceden los datos de la muestra.

Además del análisis mencionado, se consideró necesario llevar a cabo un planteamiento en términos de proporción de variabilidad explicada y no explicada. Lo anterior, debido a que permite distinguir claramente la magnitud de la relación que existe en cada dimensión de la calidad en el servicio con respecto a las variables satisfacción del cliente y lealtad del cliente.

Adicionalmente, con el objetivo de conocer la relación entre las variables satisfacción del cliente, lealtad del cliente y calidad en el servicio, así como de un conjunto de variables explicativas (dimensiones de calidad en el servicio), se realizó un análisis factorial utilizando el análisis de componentes principales y la rotación ortogonal varimax mediante el software estadístico SPSS versión 25. Antes de realizar el análisis factorial se determinó si los ítems (variables) se encontraban suficientemente interrelacionados, para lo cual se empleó la prueba de esfericidad de Bartlett y la medida de adecuación muestral Kaiser-Mayer-
Olkin (KMO). Se realizó un análisis factorial para obtener la matriz de comunalidades, varianza total explicada y la matriz de componentes rotados. A través de la matriz de comunalidades se obtuvo la proporción de varianza de las variables explicada por los factores comunes. La varianza total explicada permitió determinar el número de factores significativos en el estudio. Se utilizó la matriz de componentes rotados para obtener una estructura simple óptima, en la que cada variable participe en la menor cantidad de factores posibles, pero maximice el número de cargas altas en cada variable. Con base en las tres pruebas mencionadas se obtuvieron los nombres de los componentes a partir de la composición de las variables iniciales, y se describieron los puntajes de las cargas factoriales que conforman cada uno.

\section{RESULTADOS Y DISCUSIÓN}

Los encuestados (clientes) que respondieron el instrumento corresponden en un $56.6 \%$ al género femenino. En los datos de escolaridad destacan los niveles de licenciatura y preparatoria, con $63.6 \%$ y $20.2 \%$, respectivamente. Los niveles de secundaria y de posgrado tienen una frecuencia de 8 clientes en cada nivel (6.2 \% para cada uno) y los clientes con nivel de educación primaria representan un $3.8 \%$ de la muestra.

En la Tabla 3 se muestran los valores generados para el coeficiente alfa de Cronbach. Se observó que el valor menor corresponde a la variable lealtad del cliente, con 0.886, y para las variables de calidad en el servicio y satisfacción del cliente pertenecen los valores superiores, con 0.906 y 0.907 cada una. Las puntuaciones más altas del coeficiente se obtuvieron al evaluar individualmente las dimensiones que integran la calidad en el servicio, con valores que oscilan entre 0.913 y 0.922 . Los valores presentados del coeficiente alfa de Cronbach demuestran que existe una consistencia interna en todas las variables y en las dimensiones de la variable calidad en el servicio. Además, se comprobó que la escala del cuestionario tiene una confiabilidad importan- 
Tabla 3. Coeficiente de alfa de Cronbach.

Table 3. Cronbach's alpha coefficient.

\begin{tabular}{|l|c|c|c|}
\hline \multicolumn{1}{|c|}{ Dimensión } & $\begin{array}{c}\text { Núm. } \\
\text { ítems }\end{array}$ & \multicolumn{2}{|c|}{ Alfa de Cronbach } \\
\hline $\begin{array}{l}\text { Calidad en el } \\
\text { servicio }\end{array}$ & 22 & & 0.906 \\
\hline Tangibles & 4 & 0.918 & \\
\hline Confiabilidad & 5 & 0.913 & \\
\hline Responsabilidad & 4 & 0.919 & \\
\hline Confianza & 4 & 0.917 & \\
\hline Empatía & 5 & 0.922 & 0.907 \\
\hline $\begin{array}{l}\text { Satisfacción del } \\
\text { cliente }\end{array}$ & 3 & & 0.886 \\
\hline $\begin{array}{l}\text { Lealtad del } \\
\text { cliente }\end{array}$ & 4 & & \\
\hline
\end{tabular}

te, debido a que los valores del alfa de Cronbach son superiores a 0.700 (Hair ycol., 2014).

En la Tabla 4 se muestra la matriz de correlación basada en el coeficiente de Spearman. Al analizar la información, a nivel general, se aprecia que existe una correlación altamente significativa, positiva y fuerte entre la variable calidad en el servicio y las variables satisfacción del cliente y lealtad del cliente, con valores de $\mathrm{r}$ de 0.820 y 0.803 , respectivamente. Además, entre las variables satisfacción del cliente y lealtad del cliente se observa que mantienen una relación aún más estrecha, al obtener una $\mathrm{r}$ de 0.827 . Por su parte, en las dimensiones que integran la calidad en el servicio con respecto a la variable de satisfacción del cliente se observa, en todos los casos, la existencia de una correlación positiva, con valores de $r$ que oscilan entre 0.684 y 0.910. Esta variable presenta una relación fuerte y positiva con la dimensión de aspectos tangibles, mientras que con el resto de las dimensiones mantiene una relación moderada.

Por otro lado, al analizar el escenario de la calidad en el servicio con respecto a la variable lealtad del cliente, se identifica una tendencia general, similar a lo que se muestra en la in- terpretación de los resultados ante la variable de satisfacción del cliente. En todas las dimensiones se aprecia la existencia de una correlación positiva, con valores de $\mathrm{r}$ que oscilan entre 0.673 y 0.919 . Al igual que en la variable satisfacción del cliente, resalta la dimensión de aspectos tangibles, con una relación fuerte y positiva, y con las otras dimensiones mantienen una relación moderada.

Con apoyo de la variabilidad explicada, se confirmó para ambos casos su validez, al interpretarse que existe una incidencia de la satisfacción del cliente de $67 \%$, y de $64 \%$ en la lealtad del cliente a partir del nivel de la prestación de la calidad en el servicio (Tabla 5). Los aspectos tangibles inciden con un $83 \%$ sobre la percepción de la satisfacción del cliente. Las dimensiones de confiabilidad, confianza y empatía inciden con $58 \%, 56 \%$ y $53 \%$, respectivamente. Por su parte, la dimensión de responsabilidad es representada con el porcentaje menor de $47 \%$. En cuanto al grado de correlación sobre la lealtad del cliente, destacan igualmente los aspectos tangibles, con un $84 \%$, y las dimensiones de confianza y confiabilidad intercambian su posición en relación con su evaluación anterior, con $58 \%$ y $57 \%$, respectivamente. En la dimensión empatía se presentó una asociación de un $48 \%$, y para la dimensión de responsabilidad con $45 \%$, que es el menor valor.

La dimensión aspectos tangibles ocupa un papel estratégico en la determinación de los niveles de satisfacción del cliente y lealtad del cliente, debido a que comparten un elevado porcentaje de información y variabilidad entre sí, $83 \%$ y $84 \%$ cada una (Tabla 5). Además, la diferencia existente entre el valor más alto y bajo de ambas variables corresponde a $36 \%$ y $39 \%$, en el mismo orden. Por tanto, de acuerdo con los métodos estadísticos, se confirma que los elementos relacionados con la apariencia de las instalaciones de la empresa, del personal, del equipo, así como del material involucrado en la prestación del servicio constituyen aspectos altamente valorados en el proceso de la evaluación de los clientes. Tal 
Tabla 4. Matriz de correlación de Spearman.

Table 4. Spearman correlation coefficient.

\begin{tabular}{|c|c|c|c|c|c|c|c|c|}
\hline Variable & $\begin{array}{c}\text { Calidad } \\
\text { en el } \\
\text { servicio }\end{array}$ & $\begin{array}{l}\text { Satisfac- } \\
\text { ción del } \\
\text { cliente }\end{array}$ & $\begin{array}{c}\text { Lealtad } \\
\text { del } \\
\text { cliente }\end{array}$ & $\begin{array}{c}\text { Tangi- } \\
\text { bles }\end{array}$ & $\begin{array}{l}\text { Confia- } \\
\text { bilidad }\end{array}$ & $\begin{array}{l}\text { Respon- } \\
\text { sabilidad }\end{array}$ & $\begin{array}{l}\text { Con- } \\
\text { fianza }\end{array}$ & $\underset{\text { tía }}{\text { Empa- }}$ \\
\hline $\begin{array}{l}\text { Calidad en el } \\
\text { servicio }\end{array}$ & 1 & $0.820^{* * *}$ & $0.803^{* * *}$ & $0.891^{* *}$ & $0.918^{* *}$ & $0.892^{* *}$ & $0.874^{* * *}$ & $0.867^{* * *}$ \\
\hline $\begin{array}{l}\text { Satisfacción } \\
\text { del cliente }\end{array}$ & $0.820^{* *}$ & 1 & $0.827^{* * *}$ & $0.910^{* *}$ & $0.764^{* * *}$ & $0.684^{* *}$ & $0.751^{* *}$ & $0.731^{* * *}$ \\
\hline $\begin{array}{l}\text { Lealtad del } \\
\text { cliente }\end{array}$ & $0.803^{* *}$ & $0.827^{* *}$ & 1 & $0.919^{* *}$ & $0.756^{* *}$ & $0.673^{* *}$ & $0.763^{* *}$ & $0.690^{* * *}$ \\
\hline Tangibles & $0.891^{* *}$ & $0.910^{* *}$ & $0.919^{* * *}$ & 1 & $0.801^{* *}$ & $0.715^{* * *}$ & $0.812^{* * *}$ & $0.732^{* *}$ \\
\hline $\begin{array}{l}\text { Confiabi- } \\
\text { lidad }\end{array}$ & $0.918^{* *}$ & $0.764^{* *}$ & $0.756^{* *}$ & $0.801^{* *}$ & 1 & $0.812^{* *}$ & $0.728^{* *}$ & $0.768^{* * *}$ \\
\hline $\begin{array}{l}\text { Responsa- } \\
\text { bilidad }\end{array}$ & $0.892^{* *}$ & $0.684^{* *}$ & $0.673^{* *}$ & $0.715^{* *}$ & $0.812^{* *}$ & 1 & $0.758^{* *}$ & $0.720^{* * *}$ \\
\hline Confianza & $0.874^{* *}$ & $0.751^{* *}$ & $0.763^{* *}$ & $0.812^{* *}$ & $0.728^{* *}$ & $0.758^{* *}$ & 1 & $0.740^{* * *}$ \\
\hline Empatía & $0.867^{* * *}$ & $0.731^{* * *}$ & $0.690^{* *}$ & $0.732^{* *}$ & $0.768^{* *}$ & $0.720^{* *}$ & $0.740^{* * *}$ & 1 \\
\hline
\end{tabular}

Tabla 5. Proporción de variabilidad explicada y no explicada.

Table 5. Proportion of explained and unexplained variance.

\begin{tabular}{|l|c|c|c|c|}
\hline \multirow{2}{*}{\multicolumn{1}{|c}{ Variable }} & \multicolumn{2}{|c|}{ Variabilidad explicada } & \multicolumn{2}{c|}{ Variabilidad no explicada } \\
\cline { 2 - 5 } & $\begin{array}{c}\text { Satisfacción } \\
\text { del cliente }\end{array}$ & $\begin{array}{c}\text { Lealtad } \\
\text { del cliente }\end{array}$ & $\begin{array}{c}\text { Satisfacción } \\
\text { del cliente }\end{array}$ & $\begin{array}{c}\text { Lealtad } \\
\text { del cliente }\end{array}$ \\
\hline Calidad en el servicio & $67 \%$ & $64 \%$ & $33 \%$ & $36 \%$ \\
\hline Tangibles & $83 \%$ & $84 \%$ & $17 \%$ & $16 \%$ \\
\hline Confiabilidad & $58 \%$ & $57 \%$ & $42 \%$ & $43 \%$ \\
\hline Confianza & $56 \%$ & $58 \%$ & $44 \%$ & $42 \%$ \\
\hline Empatía & $53 \%$ & $48 \%$ & $47 \%$ & $52 \%$ \\
\hline Responsabilidad & $47 \%$ & $45 \%$ & $53 \%$ & $55 \%$ \\
\hline
\end{tabular}

pareciera que, al conceder una calificación superior sobre dichos aspectos, el cliente supone que obtiene, por añadidura, otra serie de aspectos que influyen de forma positiva en el funcionamiento de la empresa durante el proceso de la prestación del servicio.

Las variables satisfacción del cliente y lealtad del cliente, en términos de la variabilidad no explicada, se muestran en el lado derecho de la Tabla 5, identificándose que la dimensión responsabilidad presenta los porcentajes más altos, con $53 \%$ y $55 \%$ en cada variable. Existe una contundente distancia, en la proporción de variabilidad no explicada, entre los aspectos tangibles con el resto de las dimensiones. Una situación interesante radica en el hecho de que la dimensión aspectos tangibles, es la única en el instrumento utilizado, de calidad en el servicio, que pone a consideración del cliente exclusivamente situaciones tangibles. Esto es en virtud de su naturaleza, es más evi- 
dente percibir, apreciar y valorar a esta que al resto de las dimensiones.

La desproporción en los resultados, presentados en la Tabla 5, permite identificar una tendencia en el criterio utilizado por los clientes cuando se les solicita evaluar el nivel de la calidad durante la prestación de un servicio. En dicha tendencia se observó que las dimensiones están clasificadas en tres secciones, de acuerdo con el nivel de importancia asignado. En la primera sección, con un nivel de importancia alta, solamente están contemplados los aspectos tangibles. Con un grado de importancia moderada se agrupan las dimensiones de confianza, empatía y confiabilidad. Por último, con un nivel de importancia baja se encuentra la dimensión de responsabilidad, estimada por el tiempo de atención al cliente y la rapidez del servicio. Dicha situación afecta la percepción del cliente con respecto a la responsabilidad de los empleados de la empresa. Lo anterior, con base en la evaluación del cliente, constituye un aspecto relevante en la calidad en el servicio.

El comportamiento de una variable suele relacionarse con el comportamiento de un conjunto de variables (Tomaz-de-Aquino y col., 2018), permitiendo responder algunas preguntas relacionadas con el estudio planteado. Cabe mencionar que en esta sección, los 29 ítems que conforman el instrumento de investigación se denominan variables, pero siguiendo el mismo enfoque de dimensiones y aspectos relacionados con la calidad en el servicio, satisfacción del cliente y lealtad del cliente. En la Tabla 6 se presenta la prueba de esfericidad

Tabla 6. KMO y prueba Bartlett.

Table 6. KMO and Bartlett's test.

\begin{tabular}{|l|c|c|}
\hline \multicolumn{2}{|c|}{$\begin{array}{c}\text { Medida de adecuación muestral } \\
\text { de Kaiser-Meyer-Olkin }\end{array}$} & $\mathbf{0 . 9 2 0}$ \\
\hline $\begin{array}{l}\text { Prueba de } \\
\text { esfericidad de } \\
\text { Bartlett }\end{array}$ & $\begin{array}{c}\text { Chi-cuadrado } \\
\text { aproximado }\end{array}$ & 3551.977 \\
\cline { 2 - 3 } & Df & 406 \\
\cline { 2 - 3 } & Sig. & 0.000 \\
\hline
\end{tabular}

de Bartlett $(\mathrm{P}<0.000)$, con una medida de adecuación muestral de $\mathrm{KMO}=0.920$, lo que confirma que los datos son adecuados para el tamaño de la muestra. Por tanto, se puede realizar un análisis factorial (Hancock y col., 2018) para la identificación del número de componentes y evaluación de su validez.

En la Tabla 7 se muestra la matriz de comunalidades, que expresa la proporción de varianza explicada por el conjunto de factores co-

Tabla \%. Matriz de comunalidades.

Table \%. Communalities matrix.

\begin{tabular}{|c|c|c|c|}
\hline & Variable & Inicial & $\begin{array}{l}\text { Factor de } \\
\text { extracción }\end{array}$ \\
\hline 1 & TAN06 & 0.651 & 0.662 \\
\hline 2 & TANO7 & 0.680 & 0.679 \\
\hline 3 & TAN08 & 0.655 & 0.569 \\
\hline 4 & TANO9 & 0.653 & 0.622 \\
\hline 5 & CON10 & 0.692 & 0.569 \\
\hline 6 & CON11 & 0.741 & 0.638 \\
\hline 7 & CON12 & 0.474 & 0.235 \\
\hline 8 & CON13 & 0.744 & 0.661 \\
\hline 9 & CON14 & 0.664 & 0.555 \\
\hline 10 & RES15 & 0.709 & 0.531 \\
\hline 11 & RES16 & 0.778 & 0.687 \\
\hline 12 & RES17 & 0.876 & 0.826 \\
\hline 13 & RES18 & 0.871 & 0.833 \\
\hline 14 & CONF19 & 0.738 & 0.622 \\
\hline 15 & CONF20 & 0.802 & 0.749 \\
\hline 16 & CONF21 & 0.832 & 0.850 \\
\hline 17 & CONF22 & 0.838 & 0.820 \\
\hline 18 & EMP23 & 0.772 & 0.748 \\
\hline 19 & EMP24 & 0.565 & 0.464 \\
\hline 20 & EMP25 & 0.765 & 0.619 \\
\hline 21 & EMP26 & 0.702 & 0.689 \\
\hline 22 & EMP27 & 0.838 & 0.764 \\
\hline 23 & SAT28 & 0.869 & 0.824 \\
\hline 24 & SAT29 & 0.835 & 0.758 \\
\hline 25 & SAT30 & 0.847 & 0.729 \\
\hline 26 & LEA31 & 0.803 & 0.640 \\
\hline 27 & LEA32 & 0.921 & 0.903 \\
\hline 28 & LEA33 & 0.905 & 0.899 \\
\hline 29 & LEA34 & 0.593 & 0.514 \\
\hline
\end{tabular}

TAN = Tangibles; CON = Confiabilidad; RES = Responsabilidad; CONF = Confianza; EMP = Empatía; SAT $=$ Satisfacción del cliente; LEA = Lealtad del cliente. 
munes resultantes. Por un lado, las variables LEA32 y LEA33 son las mejor explicadas por el conjunto de factores retenidos; en ambos casos, la varianza explicada supera un $89 \%$. Por otro lado, CON12 y EMP24 presentan las menores proporciones de varianza explicada por los factores, con un 0.235 y 0.464 , respectivamente.
En la Tabla 8 se muestra la varianza total explicada, la cual permite realizar un análisis con mayor detalle estadístico. En la sección de la tabla "Valores propios iniciales - Initial Eigenvalues" se puede observar el "valor propio total", que es la parte de la varianza de las variables inicialmente consideradas que explican cada uno de los factores del análisis factorial

Tabla 8. Varianza total explicada.

Table 8. Total variance explained.

\begin{tabular}{|c|c|c|c|c|c|c|c|c|c|}
\hline \multirow{2}{*}{ 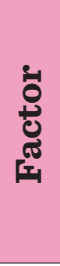 } & \multicolumn{3}{|c|}{$\begin{array}{l}\text { Valores propios iniciales } \\
\text { Initial Eigenvalues }\end{array}$} & \multicolumn{3}{|c|}{$\begin{array}{l}\text { Extracción de las sumas de } \\
\text { cargas al cuadrado }\end{array}$} & \multicolumn{3}{|c|}{$\begin{array}{l}\text { Rotación de las sumas de } \\
\text { cargas al cuadrado }\end{array}$} \\
\hline & Total & $\begin{array}{c}\% \\
\text { de la } \\
\text { varianza }\end{array}$ & $\begin{array}{c}\% \\
\text { acumulado } \\
\text { de la } \\
\text { varianza }\end{array}$ & Total & $\begin{array}{c}\% \\
\text { de la } \\
\text { varianza }\end{array}$ & $\begin{array}{c}\% \\
\text { acumulado } \\
\text { de la } \\
\text { varianza }\end{array}$ & Total & $\begin{array}{c}\% \\
\text { de la } \\
\text { varianza }\end{array}$ & $\begin{array}{c}\% \\
\text { acumulado } \\
\text { de la } \\
\text { varianza }\end{array}$ \\
\hline 1 & 15.917 & 54.886 & 54.886 & 15.535 & 53.570 & 53.570 & 4.726 & 16.296 & 16.296 \\
\hline 2 & 1.825 & 6.293 & 61.179 & 1.474 & 5.082 & 58.652 & 4.524 & 15.602 & 31.898 \\
\hline 3 & 1.391 & 4.796 & 65.975 & 1.038 & 3.578 & 62.230 & 3.765 & 12.982 & 44.880 \\
\hline 4 & 1.158 & 3.992 & 69.967 & 0.884 & 3.047 & 65.277 & 3.585 & 12.361 & 57.242 \\
\hline 5 & 1.086 & 3.746 & 73.713 & 0.728 & 2.510 & 67.787 & 3.058 & 10.545 & 67.787 \\
\hline 6 & 0.883 & 3.046 & 76.759 & & & & & & \\
\hline 7 & 0.721 & 2.488 & 79.247 & & & & & & \\
\hline 8 & 0.623 & 2.147 & 81.393 & & & & & & \\
\hline 9 & 0.619 & 2.135 & 83.528 & & & & & & \\
\hline 10 & 0.514 & 1.772 & 85.300 & & & & & & \\
\hline 11 & 0.469 & 1.617 & 86.917 & & & & & & \\
\hline 12 & 0.437 & 1.506 & 88.423 & & & & & & \\
\hline 13 & 0.428 & 1.474 & 89.897 & & & & & & \\
\hline 14 & 0.353 & 1.216 & 91.113 & & & & & & \\
\hline 15 & 0.311 & 1.071 & 92.184 & & & & & & \\
\hline 16 & 0.283 & 0.974 & 93.158 & & & & & & \\
\hline 17 & 0.258 & 0.891 & 94.049 & & & & & & \\
\hline 18 & 0.244 & 0.841 & 94.890 & & & & & & \\
\hline 19 & 0.230 & 0.792 & 95.681 & & & & & & \\
\hline 20 & 0.208 & 0.718 & 96.399 & & & & & & \\
\hline 21 & 0.195 & 0.671 & 97.070 & & & & & & \\
\hline 22 & 0.169 & 0.583 & 97.653 & & & & & & \\
\hline 23 & 0.150 & 0.517 & 98.170 & & & & & & \\
\hline 24 & 0.139 & 0.478 & 98.648 & & & & & & \\
\hline 25 & 0.118 & 0.405 & 99.053 & & & & & & \\
\hline 26 & 0.094 & 0.326 & 99.379 & & & & & & \\
\hline 27 & 0.078 & 0.268 & 99.647 & & & & & & \\
\hline 28 & 0.066 & 0.227 & 99.873 & & & & & & \\
\hline 29 & 0.037 & 0.127 & 100.00 & & & & & & \\
\hline
\end{tabular}


exploratorio. El valor propio expresa la cantidad de la varianza total que está explicada por un factor, por ejemplo, el "Factor 1" de la Tabla 8 expresa 15.917 de varianza, lo cual representa un $54.886 \%$ de la varianza total. El valor propio es superior a 1.000 en los 5 primeros casos, lo que significa que, cada uno de estos factores explicaría la varianza de más de una variable del estudio. Es decir, el análisis asume que las 29 variables originales se pueden reducir a 5 factores subyacentes. También se puede apreciar que el "porcentaje de la varianza" alcanza su máximo valor en el primer factor, es decir, este factor explica $54.886 \%$ de la varianza total del modelo; y del segundo al quinto factor la varianza acumulada alcanza un $73.713 \%$. Es decir, cuando se supone que existen estos 5 factores, se puede predecir el $73.713 \%$ de la información en las 29 variables. Es de notarse que los factores en la Tabla 8 se ordenan de manera descendente, donde se observa que la varianza del modelo es menos explicada por los factores que se ubican en la parte final.

La matriz de componentes rotados, que se muestra en la Tabla 9, permite una mayor discriminación de las variables con respecto a los componentes. El proceso de rotación facilita la interpretación de las asociaciones entre variables y los componentes, al permitir que las variables fuertemente correlacionadas presenten pesos factoriales elevados y las variables con una correlación menor obtengan pesos factoriales bajos. El resultado contenido en la matriz de componentes rotados condujo a 28 variables (se eliminó la variable CON12) con una extracción de 5 componentes (coincidente con la varianza total explicada) convergiendo en 10 iteraciones. También se observa en la Tabla 9 que la mayoría de las variables del componente 1 muestran pesos factoriales superiores a 0.500, con excepción de las variables CON11, CON14 y LEA34, que registran un valor ligeramente inferior. En el componente 2 (Tabla 9), las variables presentan pesos factoriales elevados en todos los casos, destacando la variable CONF21, con un peso de 0.805. Este comportamiento es similar para los componentes 3,
Tabla 9. Matriz de componentes rotados.

Table 9. Rotated component matrix.

\begin{tabular}{|c|c|c|c|c|c|c|}
\hline & & & Con & iponer & te & \\
\hline & Variable & 1 & 2 & 3 & 4 & 5 \\
\hline 1 & RES17 & 0.692 & & & & \\
\hline 2 & RES16 & 0.687 & & & & \\
\hline 3 & CON13 & 0.634 & & & & \\
\hline 4 & RES18 & 0.633 & & & & \\
\hline 5 & CON10 & 0.587 & & & & \\
\hline 6 & RES15 & 0.574 & & & & \\
\hline 7 & CON11 & 0.465 & & & & \\
\hline 8 & CON14 & 0.435 & & & & \\
\hline 9 & LEA34 & 0.433 & & & & \\
\hline 10 & CONF21 & & 0.805 & & & \\
\hline 11 & CONF22 & & 0.706 & & & \\
\hline 12 & CONF20 & & 0.615 & & & \\
\hline 13 & CONF19 & & 0.599 & & & \\
\hline 14 & EMP27 & & 0.567 & & & \\
\hline 15 & EMP25 & & 0.544 & & & \\
\hline 16 & LEA33 & & & 0.739 & & \\
\hline 17 & LEA32 & & & 0.714 & & \\
\hline 18 & SAT28 & & & 0.624 & & \\
\hline 19 & LEA31 & & & 0.578 & & \\
\hline 20 & SAT29 & & & 0.535 & & \\
\hline 21 & EMP26 & & & & 0.669 & \\
\hline 22 & EMP23 & & & & 0.664 & \\
\hline 23 & EMP24 & & & & 0.534 & \\
\hline 24 & SAT30 & & & & 0.514 & \\
\hline 25 & TAN6 & & & & & 0.727 \\
\hline 26 & TAN7 & & & & & 0.701 \\
\hline 27 & TAN9 & & & & & 0.655 \\
\hline 28 & TAN8 & & & & & 0.522 \\
\hline \multicolumn{7}{|c|}{$\begin{array}{l}\text { Método de extracción: Máxima verosimilitud } \\
\text { Método de rotación: Varimax con } \\
\text { normalización Kaiser }\end{array}$} \\
\hline \multicolumn{7}{|c|}{ a. Rotación convergente en 10 iteraciones. } \\
\hline
\end{tabular}

TAN = Tangibles; CON = Confiabilidad; RES = Responsabilidad; CONF = Confianza; EMP = Empatía; SAT = Satisfacción del cliente; LEA = Lealtad del cliente.

4 y 5 , con valores de pesos factoriales elevados y únicamente con variaciones en la cantidad de variables que componen el componente. La variable CON12 no se incluye en la matriz de componentes rotados, debido a que no converge en ningún componente. Esta va- 
riable obtiene una carga o peso factorial de 0.304, el cual es un valor alejado del resto de los componentes. Además, el punto de corte mínimo recomendado en el análisis factorial es una carga de 0.40 (Yong y Pearce, 2013). Con el fin de confirmar la no convergencia de la variable CON12, se ejecutó por segunda ocasión el análisis factorial considerando 28 variables (excluyendo la variable CON12), obteniendo un resultado similar en la matriz de componentes rotados con 5 componentes conformados por las mismas variables, carga factorial y convergencia en 10 iteraciones, lo cual valida que la variable CON12 no afecta en la convergencia de los componentes.

La rotación realizada permite incrementar de manera óptima las saturaciones de las variables sobre los componentes. Entonces, se puede inferir la existencia de cinco patrones subyacentes al conjunto de 29 variables (ítems) introducidos inicialmente en el análisis factorial exploratorio, mismos que pueden exponerse de la siguiente forma:

Componente 1. Denominado responsabilidadconfiabilidad, porque agrupa a todas las variables de la dimensión responsabilidad y $80 \%$ de las variables de la dimensión confiabilidad. Lo que indica que el cliente percibe la responsabilidad que los empleados transmiten y la vincula con la confiabilidad del servicio prestado.

Componente 2. Identificado como confianzaempatía, debido a que se compone de todas las variables de la dimensión confianza y $40 \%$ de las variables de la dimensión empatía. La característica de este componente se centra en que las variables EMP25 y EMP27 están fuertemente relacionadas con las variables de la dimensión confianza. Lo anterior, en virtud de que buscan determinar si el cliente identifica en los empleados las habilidades y profesionalismo con que lo atienden, en relación con el servicio requerido.

Componente 3. Llamado lealtad-satisfacción, se conforma por $75 \%$ de las variables de leal- tad del cliente y por $67 \%$ de satisfacción del cliente. Los indicadores de este componente tienen que ver con la intención de que el cliente regrese a adquirir un producto o servicio a la empresa, así como recomendar $\mathrm{y}$ hacer comentarios positivos de la misma. Además de la satisfacción por el servicio o producto adquirido, las expectativas fueron superadas mediante el servicio.

Componente 4. Denominado empatía-satisfacción, se conforma por $60 \%$ de las variables de la dimensión empatía y por $33 \%$ de las variables de satisfacción del cliente. Se relaciona con la empatía que muestran los empleados hacia los clientes durante la prestación del servicio, aspectos tales como asistencia personalizada, horarios de atención, interés por atender al cliente o por superar el nivel de servicio esperado.

Componente 5. Identificado como tangibles, debido a que únicamente se integra por las cuatro variables de la dimensión de aspectos tangibles. Este componente se relaciona con el aspecto visual que el cliente identifica en la empresa, destacando las instalaciones o infraestructura, equipo y tecnología utilizados para prestar el servicio, así como la formalidad y vestimenta de los empleados.

En este sentido, al revisar el coeficiente de correlación entre las variables responsabilidadconfiabilidad (Tabla 4) se observó una relación altamente significativa, positiva y fuerte, con un valor de $\mathrm{r}$ de 0.812. En forma similar, la asociación entre las variables lealtad-satisfacción, con un valor de $\mathrm{r}$ de $0.82 \%$. Con respecto al coeficiente de correlación entre las variables confianza-empatía, se identificó un valor de $\mathrm{r}$ de 0.740. También en la asociación empatía-satisfacción se aprecia una relación positiva alta, con un valor de r de 0.731 .

Lo anterior es coincidente con la existencia de cinco factores críticos identificados en la varianza total explicada y en la matriz de componentes rotados. Estos resultados se pueden considerar como un punto de referencia para 
evaluar el grado de competitividad en términos del servicio proporcionado por las pequeñas y medianas empresas a sus clientes, considerando las limitaciones del estudio presentado y el contexto de aplicación.

Los resultados obtenidos se pueden comparar con trabajos previos del tema de investigación. Ibarra-Morales y Casas-Medina (2015) midieron la calidad en el servicio otorgado, a través de la percepción de los clientes. Aplicaron un instrumento basado en el modelo Servperf, considerando las 5 dimensiones, con valores en el coeficiente alfa de Cronbach entre $0.806 \mathrm{y}$ 0.898 , con una varianza total explicada utilizando el método varimax, de $74.49 \%$ en el primer factor. En el presente estudio los valores de alfa de Cronbach fueron más altos, entre 0.913 y 0.922 ; y la varianza total explicada fue de $54.88 \%$ en el primer factor. No obstante que la varianza total explicada de este trabajo es inferior a la reportada por los autores aludidos, en el segundo factor alcanza un $61.17 \%$, lo cual supera el criterio mínimo (60\%) recomendado en la literatura (Hair y col., 2014), con un porcentaje de varianza total explicada de $73.71 \%$ en cinco factores con valor propio total superior a 1 . Con respecto al coeficiente de correlación calculado para las 5 dimensiones, los resultados de este estudio concuerdan con Ibarra-Morales y Casas-Medina (2015) en la asociación entre las dimensiones aspectos tangibles, confiabilidad, responsabilidad y confianza. Sin embargo, la correlación entre empatía y confiabilidad observada en dicho trabajo no coincide con la relación fuerte identificada entre las variables de empatía y confianza en la presente investigación. Estos autores también observaron que las dimensiones aspectos tangibles, confianza y empatía intervienen en un $81.4 \%$ en la satisfacción del cliente pero las dimensiones de confiabilidad y responsabilidad afectan en forma considerable la percepción del cliente con respecto al servicio prestado por la empresa. La dimensión de confiabilidad es la que menos aporta a la satisfacción del cliente, con un $76 \%$, relacionada con el interés que los empleados muestran para realizar su trabajo en forma rápida y preci- sa, así como la aptitud para resolver los problemas expuestos por los clientes. En el presente trabajo la dimensión tangible contribuye un $83 \%$ en la satisfacción del cliente, porcentaje similar al de la lealtad del cliente, mientras que la dimensión de responsabilidad es la que menos aporta a la satisfacción del cliente, con un $47 \%$.

Por otro lado, Kumar y Hundal (2019), mediante un análisis factorial, obtuvieron en su investigación un valor del coeficiente de alfa de Cronbach de 0.827, considerando los 22 ítems del enfoque Servqual para medir la calidad en el servicio de una empresa de energía solar, cuyos principales clientes son de zonas rurales; y reportaron un valor de 0.842 en la medida de adecuación muestral KMO. Los dos valores fueron más bajos que los del presente estudio. Además, la varianza total explicada se conforma de 5 factores con un valor de 82.74 \%, donde el primer factor alcanza solo un $25.56 \%$ de la varianza total y se requieren de 3 factores para superar el $50 \%$ de la varianza total explicada. En el presente trabajo, el valor de la varianza explicada de el primer factor explica una cantidad significativamente mayor de la varianza. Este porcentaje acumulado de la varianza explicada en el primer factor sugiere un buen ajuste de la solución del componente. Por otro lado, los autores mencionados, también encontraron en su investigación que la brecha entre la expectativa y la percepción del cliente es mínima en las dimensiones de confiabilidad, responsabilidad, confianza y empatía; por ejemplo, en la dimensión de responsabilidad se encontró un $80.44 \%$ de satisfacción del cliente, por la capacidad de respuesta que encontraron en la empresa. Sin embargo, en la dimensión de aspectos tangibles observaron una brecha muy alta entre la expectativa y percepción, solo un $56.66 \%$ de los clientes percibe que se cumplen expectativas. Los ítems que se incluyeron en el instrumento para evaluar la dimensión de tangibilidad se relacionan con el diseño moderno de los productos de energía solar, el estado de las instalaciones de la empresa, la funcionalidad de las instalaciones del 
área de ventas y atención a clientes, la variedad de productos y el rendimiento de los productos solares. También, en la matriz de componentes rotados, estos autores utilizaron un método de extracción de análisis de componentes principales; los componentes se conforman por las variables de cada dimensión con cargas factoriales superiores a 0.6 en el siguiente orden: confiabilidad, responsabilidad, confianza, empatía y tangibles. Los resultados obtenidos en la matriz de componentes rotados coinciden con el presente trabajo en la conformación del cuarto y del quinto componente.

Lai y Nguyen (2017) realizaron un análisis factorial similar al de este trabajo, pero aplicado en un estudio para evaluar la calidad en el servicio de los proveedores de telecomunicaciones. Los resultados estadísticos siguen la misma tendencia que los de la presente investigación, pero con un porcentaje acumulado de la varianza menor en los primeros cinco casos. En forma similar, concluyen que la calidad del servicio impactó positivamente en la satisfacción del cliente, toda vez que las dimensiones de empatía, confianza y aspectos tangibles fueron las que influyeron fuertemente en un $75.33 \%$, en la satisfacción del cliente. Lo anterior indica que cuando las compañías de telefonía móvil brindan una buena calidad de servicio, la satisfacción del cliente puede mejorarse. Por otro lado, las dimensiones de confiabilidad y responsabilidad tienen una percepción muy baja (69 \%) con respecto a las expectativas del cliente.

En el presente estudio se pudo comprobar que los clientes que evaluaron la calidad en el servicio de la empresa papelera analizada se sintieron satisfechos y mostraron lealtad a la pyme, al percibir buena atención, por lo que se puede sugerir que la rentabilidad y sostenibilidad de la empresa dependen de la calidad del servicio.

Las limitaciones de este trabajo incluyen el sesgo de los encuestados, que puede afectar el resultado en lo que respecta a los datos colectados. También, que la encuesta se aplicó solamente en el edificio matriz de la empresa (establecimiento con el volumen de ventas más alto dentro de la organización). Debido a los sesgos de los encuestados, los resultados pueden no ser los mismos para todos los demás establecimientos (sucursales) de la empresa. Además, la encuesta se realizó únicamente en Ciudad Victoria y el tamaño de la muestra es solo de 129 encuestados, por lo que es posible que no refleje un panorama más amplio. En un estudio futuro es recomendable considerar no solo la venta directa en mostrador, también se deben incluir los clientes (normalmente empresas) que efectúan compras mediante el servicio por teléfono o por sistemas de comercio electrónico con entrega a domicilio, así como las ventas a distribuidores y no solo las directas al público. Por otro lado, es importante considerar el giro comercial, dedicado a la venta de artículos de oficina y papelería, clasificada en una estratificación de mediana empresa dentro las pymes, de acuerdo con el número de empleados y al volumen de ventas anuales. Los resultados se pueden tomar como referencia para futuras investigaciones, pero pueden variar por el giro de la empresa y el sector (manufacturas, servicios o comercio) al que pertenezca dentro la clasificación de las pymes.

\section{CONCLUSIONES}

Se confirmó que la calidad en el servicio se asocia positivamente con las variables de satisfacción del cliente y lealtad del cliente. Por su nivel de importancia, se clasifican en orden de mayor a menor en responsabilidad-confiabilidad, confianza-empatía, lealtad-satisfacción, empatía-satisfacción y tangibles. Además, se confirma una correlación fuerte en la variable de la calidad en el servicio, en primera instancia, entre los ítems correspondientes a las dimensiones responsabilidad y confiabilidad. También se observa este comportamiento entre los ítems de las dimensiones de confianza y empatía. De igual manera, los aspectos tangibles se concentran en un solo componente. Finalmente, los resultados del estudio presentado sugieren que las dimensiones aspectos tangibles, confiabilidad y confianza de la calidad en 
el servicio tienen una relación significativa positiva con la satisfacción del cliente y en la variación de la percepción del mismo con respecto al servicio recibido.

\section{REFERENCIAS}

ACSI, American Customer Satisfaction Index (2018). Customer satisfaction reports. American Customer Satisfaction Index. [En línea]. Disponible en: https://www.theacsi.org/news-and -resources/customer-satisfaction-reports/reports-2018. Fecha de consulta: 9 de septiembre de 2018 .

Ahrholdt, D. C., Gudergan, S. P., and Ringle, C. M. (2017). Enhancing service loyalty: The roles of delight, satisfaction, and service quality. Journal of Travel Research. 56(4): 436-450.

Azman, S. and Gomiscek, B. (2015). Functional form of connections between perceived service quality, customer satisfaction and customer loyalty in the automotive servicing industry. Total Quality Management. 26(8): 888-904.

Azman, I. and Yusrizal, S. (2016). Service quality as a predictor of satisfaction and customer loyalty. Scientific Journal of Logistics. 12(4): 269-283.

Aznar, J. P., Bagur, L., and Rocafort, A. (2016). Impact of service quality on competitiveness and profitability: The hotel industry in the Catalan coast. Intangible Capital. 12(1): 147-166.

Banwo, A. O., Du, J., and Onokala, U. (2017). The determinants of location specific choice: small and medium-sized enterprises in developing countries. Journal of Global Entrepreneurship Research. 7(16): 1-17.

BLS, Bureau of Labor Statistics (2016). Business employment dynamics - Enterpreneurship and the U.S. economy. [En línea]. Disponible en: https://www.bls.gov/bdm/entrepreneurship/ entrepreneurship.htm. Fecha de consulta: 21 de mayo de 2018.

Blut, M., Frennea, C. M., Mittal, V., and Mothersbaugh, D. L. (2015). How procedural, financial and relational switching costs affect customer satisfaction, repurchase intentions, and repurchase behavior: A meta-analysis. International Journal of Research in Marketing. 32(2):226-229.

Bustamante, J. C. (2015). Uso de variables mediadoras y moderadoras en la explicación de la lealtad del consumidor en ambientes de servicios. Estudios Gerenciales. 31(136): 299-309.

Cronin, J., Morris, M. H., and Taylor, S. (1994). Servperf vs. Servqual: reconciling performance based and perceptions minus expectations measurement of service quality. Journal of Marketing. 58(1): 125-131.

El-Adly, M. I. (2019). Modelling the relationship between hotel perceived value, customer satisfaction, and customer loyalty. Journal of Retailing and Consumer Services. 50: 322-332.

Ferreira-de-Araújo, P., Crema, M., and Verbano, C. (2019). Risk management in SMEs: A systematic literature review and future directions, in European Management Journal. [En línea]. Disponible en: https://www.sciencedirect.com/science/ article/abs/pii/S0263237319300751. Fecha de consulta: 24 de agosto de 2019.

Fourie, L. (2015). Customer satisfaction: a key to survival for SMEs? Problems and Perspectives in Management. 13(3): 181-188.

Guatzozón, M., Canto, A. M. y Pereyra, A. (2020). Calidad en el servicio en micronegocios del sector artesanal de madera en una comisaría de Mérida, México. Ingeniare: Revista Chilena de Ingeniería. 28(1):120-132.

Guesalaga, R. and Pitta, D. (2014). The importance and formalization of service quality dimensions: a comparison of Chile and the USA. Journal of Consumer Marketing. 31(2):145-151.

Hair, J. F., Black, W. C., Babin, B. J., and Anderson, R. E. (2014). Multivariate Analysis Data. (Seventh edition). USA: Pearson. 739 Pp.

Hancock, G. R., Stapleton, L. M., and Mueller, R. O. (2018). The reviewer's guide to quantitative methods in the social sciences (Second edition). USA: Taylor \& Francis Group. 514 Pp.

Ibarra-Morales, E. y Casas-Medina, E. V. (2015). Aplicación del modelo Servperf en los centros de atención Telcel, Hermosillo: una medición de la calidad en el servicio. Contaduría y Administración. 60(1):229-260.

INEGI, Instituto Nacional de Estadística y Geografía (2015). Esperanza de vida de los negocios, en Boletín 08\%/15. [En línea]. Disponible en: http://www.inegi.org.mx/saladeprensa/bole tines/2015/especiales/especiales2015_02_38.pdf. Fecha de consulta: 21 de mayo de 2018.

INEGI, Instituto Nacional de Estadística y Geografía (2019). Encuesta Nacional sobre Productividad y Competitividad de las Micro, Pequeñas y Medianas Empresas (ENAPROCE), en Secretaría de Economía. [En línea]. Disponible en: https://www.inegi. org.mx/contenidos/saladeprensa/boletines/2019/especiales /ENAPROCE2018.pdf. Fecha de consulta: 12 de octubre de 2019.

Jain, P. and Aggarwal, V. S. (2017). The effect of perceived service quality on customer satisfaction and customer loyalty in organized retail chains. Amity Business Review. 18(2): 77-89.

Kasiri, L. A., Guan-Cheng, K. T., Sambasivan, M., and Sidin, S. M. (2017). Integration of standardization and customization: Impact on service quality, customer satisfaction, and loyalty. Journal of Retailing and Consumer Services. 35: 91-97.

Kumar, V. and Hundal, B. S. (2019). Evaluating the service quality of solar product companies using SERVQUAL model. International Journal of Energy Sector Management. 13(3): 670-693. 
Lai, C. and Nguyen, M. (2017). Factors affecting service quality, customer satisfaction and loyalty of mobile phone service providers in Vietnam. International Journal of Organizational Innovation. 10(2): 75-85.

López, L. I. y Díaz, J. D. (2012). Propuesta metodológica para la gestión del cliente. Mercados y Negocios. 25(13): 5-20.

Malhotra, N. K., Agarwal, J., and Shainesh, G. (2018). Does country or culture matter in global marketing? An empirical investigation of service quality and satisfaction model with moderators in three countries. In J. Agarwal and T. Wu (Eds.), Emerging Issues in Global Marketing (pp. 61-91). Switzerland: Springer.

Michna, A. (2018). The mediating role of firm innovativeness in the relationship between knowledge sharing and customer satisfaction in SMEs. Engineering Economics 29(1): 93-103.

Murali, S., Pugazhendhi, S., and Muralidharan, C. (2016). Modelling and investigating the relationship of after sales service quality with customer satisfaction, retention and loyalty - A case study of home appliances business. Journal of Retailing and Consumer Services. 30(1): 67-83.

Nyadzayo, M. W. and Knajehzadeh, S. (2016). The antecedents of customer loyalty: A moderated mediation model of customer relationship management quality and brand image. Journal of Retailing and Consumer Services. 30(1): 262-270.

OCDE, Organización para la Cooperación y Desarrollo Económico (2018). Declaration on strengthening SMEs and entrepreneurship for productivity and inclusive growth, in OECD $\mathrm{Mi}$ nisterial Conference on SMEs. Mexico City. [En línea]. Disponible en: https://www.oecd.org/cfe/smes/ministerial/SME-Ministe rial-Declaration-ENG.pdf. Fecha de consulta: 17 de marzo de 2019.

Palese, B. and Usai, A. (2018). The relative importance of service quality dimensions in E-commerce experiences. International Journal of Information Management. 40:132-140.

Parasuraman, A., Zeithaml, V. A., and Berry, L. L. (1988). Servqual: a multiple-item scale for measuring consumer perceptions of service quality. Journal of Retailing. 64(1): 12-40.

Saleem, S. S., Moosa, K., Imam, A., and Khan, R. A. (2017). Service quality and student satisfaction: The moderating role of university culture, reputation and Price in education sector of Pakistan. Iranian Journal of Management Studies. 10(1): 237-258.

Salinas-Reyes, M., Badillo-Piña, I. y Tejeida-Padilla, R. (2018). Determinantes sistémicos de viabilidad en las pequeñas y medianas empresas (PYMES) en México. Científica. 22(2): 147-156.

Sandada, M. and Matibiri, B. (2016). An investigation into the impact of service quality, frequent flier programs and safety perception on satisfaction and customer loyalty in the airline industry in Southern Africa. South East European Journal of Economics and Business. 11(1): 41-53.

Sarmiento, R., Whelan, G., and Sprenger, J. (2018). Estudios de caso y la falsificación Popperiana: una nota de investigación sobre el artículo de Flyvbjerd titulado "Cinco malentendidos acerca de la investigación mediante los estudios de caso". Forum: Qualitative Social Research. 19(1): 14.

SBA, Small Business Administration (2017). Small business GDP. The United States Small Business Administration. [En línea]. Disponible en: https://www.sba.gov/. Fecha de consulta: 17 de marzo de 2018.

SE, Secretaría de Economía (2009). Acuerdo por el que se establece la estratificación de las micro, pequeñas y medianas empresa, en Marco Normativo. [En línea]. Disponible en: https:/ /www.economia.gob.mx/files/marco_normativo/A539.pdf. Fecha de consulta: 9 de septiembre de 2020.

Shah, S. N. U. and Baloch, Q. B. (2017). Comparative analysis on service quality \& customer satisfaction: Empirical evidence from Pakistan hotel industry. Journal of Business and Tourism. 3(2): 159-174.

Shah, S. N. U., Jan, S., and Baloch, Q. B. (2018). Role of service quality and customer satisfaction in firm's performance: Evidence from Pakistan hotel industry. Journal of Commerce and Social Sciences. 12(1): 167-182.

Shi, Y., Prentice, C., and He, W. (2014). Linking service quality, customer satisfaction and loyalty in casinos, does membership matter. International Journal of Hospitality Management. 40: 81-91.

Srivastava, M. and Rai, A. K. (2013). Investigating the mediating effect of customer satisfaction in the service quality customer loyalty relationship. Journal of Consumer Satisfaction, Dissatisfaction \& Complaining Behavior. 26(3): 95-109.

Srivastava, M. and Rai, A. K. (2018). Mechanics of engendering customer loyalty: A conceptual framework. IIMB $M a$ nagement Review. 30(3): 207-218.

Tomaz-de-Aquino, J., Valença-de-Souza, J., Lima-da-Silva, V., Jerônimo, T., and Melo, F. (2018). Factors that influence the quality of services provided by the bus rapid transit system. Benchmarking: An International Journal. 25(9): 4035-4057.

Usman, M., Shafiq, M., Savino, M., Rashid, Z., Yehya, M. I., Naqvi, A. A., ..., and Menanno, M. (2019). Investigating the role of QMS implementation on customers' satisfaction: A case study of SMEs. IFAC-PapersOnLine. 52(13): 2032-2037.

Yin, R. K. (2013). Case study research design and methods. (Fifth edition). California: SAGE publications. $282 \mathrm{Pp}$.

Yong, A. G. and Pearce, S. (2013). A beginner's guide to factor analysis: Focusing on exploratory factor analysis. Tutorials in Quantitative Methods for Psychology. 9(2): 79-94.

Zhang, S. and Hou, Y. (2013). A SERVQUAL model for assessment of service quality in supply chain. Information Technology Journal. 12(15): 3472-3475. 